\title{
On the Hill's Operator with Matrix Potential
}

\author{
O.A.Veliev \\ Depart. of Math, Fac.of Arts and Sci., \\ Dogus University, Acıbadem, 34722, \\ Kadiköy, Istanbul, Turkey. \\ e-mail: oveliev@dogus.edu.tr
}

\begin{abstract}
In this article we obtain the asymptotic formulas for the eigenvalues and eigenfunctions of the self-adjoint operator generated by a system of Sturm-Liouville equations with summable coefficients and the quasiperiodic boundary conditions. Then using these asymptotic formulas, we find the conditions on the potential for which the number of gaps in the spectrum of the Hill's operator with matrix potential is finite.
\end{abstract}

Let $L(Q(x))$ be the differential operator generated in the space $L_{2}^{m}(-\infty, \infty)$ of the vector functions by the differential expression

$$
-y^{\prime \prime}(x)+Q(x) y(x)
$$

where $Q(x)=\left(b_{i, j}(x)\right)$ is a $m \times m$ Hermitian matrix with the complex-valued summable entries $b_{i, j}(x)$ and $Q(x+1)=Q(x)$. It is well-known that the spectrum of the operator $L$ is the union of the spectra of the operators $L_{t}$ for $t \in[0,2 \pi)$ generated in $L_{2}^{m}(0,1)$ by the differential expression (1) and the quasiperiodic conditions

$$
y^{\prime}(1)=e^{i t} y^{\prime}(0), y(1)=e^{i t} y(0) .
$$

Note that $L_{2}^{m}(a, b)$ is the set of the vector functions

$f(x)=\left(f_{1}(x), f_{2}(x), \ldots, f_{m}(x)\right)$ with $f_{k}(x) \in L_{2}(a, b)$ for $k=1,2, \ldots, m$. The norm $\|$.$\| and inner product (.,$.$) in L_{2}^{m}(a, b)$ are defined by

$$
\|f\|=\left(\int_{a}^{b}|f(x)|^{2} d x\right)^{\frac{1}{2}},(f(x), g(x))=\int_{a}^{b}\langle f(x), g(x)\rangle d x,
$$

where $|$.$| and \langle.,$.$\rangle are the norm and inner product in \mathbb{C}^{m}$.

Let us introduce some preliminary results and describe briefly the scheme of the paper. Clearly, 


$$
\varphi_{k, 1, t}=\left(\begin{array}{c}
e^{i(2 \pi k+t) x} \\
0 \\
\vdots \\
0
\end{array}\right), \varphi_{k, 2, t}=\left(\begin{array}{c}
0 \\
e^{i(2 \pi k+t) x} \\
\vdots \\
0
\end{array}\right), \ldots, \varphi_{k, m, t}=\left(\begin{array}{c}
0 \\
\vdots \\
0 \\
e^{i(2 \pi k+t) x}
\end{array}\right)
$$

are the eigenfunctions of the operator $L_{t}(0)$ corresponding to the eigenvalue $(2 \pi k+t)^{2}$. If $t \neq 0, \pi$ then the multiplicity of the eigenvalue $(2 \pi k+t)^{2}$ is $m$ and the corresponding eigenspace is $E_{k}(t)=\operatorname{span}\left\{\varphi_{k, 1, t}, \varphi_{k, 2, t}, \ldots, \varphi_{k, m, t}\right\}$. In the cases $t=0$ and $t=\pi$ the multiplicity of the eigenvalues $(2 \pi k)^{2}$ and $(2 \pi k+\pi)^{2}$ is $2 m$ and the corresponding eigenspaces are

$E_{k}(0)=\operatorname{span}\left\{\varphi_{n, j, 0}: n=k,-k ; j=1,2, \ldots m\right\}$ and

$E_{k}(\pi)=\operatorname{span}\left\{\varphi_{n, j, \pi}: n=k,-(k+1) ; j=1,2, \ldots m\right\}$ respectively

It easily follows from the classical investigations [1] that the eigenvalues of the operator $L_{t}(Q)$ consist of $m$ sequences

$$
\left\{\lambda_{k, 1}(t): k \in \mathbb{Z}\right\},\left\{\lambda_{k, 2}(t): k \in \mathbb{Z}\right\}, \ldots,\left\{\lambda_{k, m}(t): k \in \mathbb{Z}\right\}
$$

satisfying the following, uniform with respect to $t \in[0,2 \pi)$, asymptotic formulas

$$
\lambda_{k, j}(t)=(2 \pi k+t)^{2}+O\left(k^{1-\frac{1}{2 m}}\right)
$$

for $j=1,2, \ldots, m$. We say that the formula $f(k, t)=O(h(k))$ is uniform with respect to $t \in[0,2 \pi)$ if there exists a positive constant $c$, independent on $t$, such that $\mid f(k, t))|<c| h(k) \mid$ for all $t \in[0,2 \pi)$ and $k \in \mathbb{Z}$.

In forthcoming relations by $N$ we denote a big positive integer, that is, $N \gg 1$, and by $c_{k}$ for $k=1,2, \ldots$, the positive constants, independent on $N$ and $t$, whose exact values are inessential. The formula (4) shows that the eigenvalue $\lambda_{k, j}(t)$ of $L_{t}(Q)$ is close to the eigenvalue $(2 k \pi+t)^{2}$ of $L_{t}(Q)$, namely :

$$
\left|\lambda_{k, j}(t)-(2 k \pi+t)^{2}\right|<c_{1}|k|^{1-\frac{1}{2 m}} .
$$

To analyze the distance of the eigenvalue $(2 k \pi+t)^{2}$ of $L_{t}(0)$ from the other eigenvalue $(2 p \pi+t)^{2}$ of $L_{t}(Q)$, which is important in perturbation theory, we have to consider three cases.

Case $1: t \in T(k)$, where

$T(k)=\left[-\frac{\pi}{2}, \frac{3 \pi}{2}\right) \backslash\left(\left(-(\ln |k|)^{-1},(\ln |k|)^{-1}\right) \cup\left(\pi-(\ln |k|)^{-1}, \pi+(\ln |k|)^{-1}\right)\right)$,

Case 2: $t \in\left(\left(-(\ln |k|)^{-1},(\ln |k|)^{-1}\right)\right.$,

Case 3: $\left.t \in\left(\pi-(\ln |k|)^{-1}, \pi+(\ln |k|)^{-1}\right)\right)$.

Using (4) one can easily verify that the inequalities

$$
\begin{aligned}
\left|(2 k \pi+t)^{2}-(2 \pi p+t)^{2}\right| & >c_{2}(\ln |k|)^{-1}(|| k|-| p||+1)(|k|+|p|), \\
\left|\lambda_{k, j}(t)-(2 \pi p+t)^{2}\right| & >c_{2}(\ln |k|)^{-1}(|| k|-| p||+1)(|k|+|p|)
\end{aligned}
$$

hold in Case 1, Case 2, and Case 3 for $p \neq k$, for $p \neq k,-k$ and for

$p \neq k,-(k+1)$ respectively. To avoid the listing of these cases repeatedly we introduce the following notation

$$
A(k, t)=\left\{\begin{array}{l}
\{k\} \text { for } t \in T(k), \\
\left.\{k,-k\} \text { for } t \in(-\ln |k|)^{-1},(\ln |k|)^{-1}\right), \\
\{k,-k-1\} \text { for } t \in\left(\pi-(\ln |k|)^{-1}, \pi+(\ln |k|)^{-1}\right)
\end{array}\right\} .
$$


Thus the inequalities (5) hold for $p \notin A(k, t)$. This implies the following purposive relations

$$
\begin{gathered}
\sum_{p: p>d} \frac{1}{\left|\lambda_{k, j}(t)-(2 \pi p+t)^{2}\right|}<\frac{c_{3}}{d}, \forall d>2|k|, \\
\sum_{p: p \notin A(k, t)} \frac{1}{\left|\lambda_{k, j}(t)-(2 \pi p+t)^{2}\right|}=O\left(\frac{\ln |k|}{k}\right), \\
\sum_{p: p \notin A(k, t)} \frac{1}{\left|\lambda_{k, j}(t)-(2 \pi p+t)^{2}\right|^{2}}=O\left(\frac{(\ln |k|)^{2}}{k^{2}}\right) \\
\sum_{p: p \notin A(k, t)} \frac{1}{\left|\lambda_{p, j}(t)-(2 \pi k+t)^{2}\right|^{2}}=O\left(\frac{(\ln |k|)^{2}}{k^{2}}\right)
\end{gathered}
$$

The formulas (7)-(10) are uniform with respect to $t \in\left[-\frac{\pi}{2}, \frac{3 \pi}{2}\right)$.

In this paper we suggest a method by which the asymptotic formulas of high accuracy for the eigenvalues $\lambda_{k, j}(t)$ and for the corresponding normalized eigenfunctions $\Psi_{k, j, t}(x)$ of $L_{t}(Q)$ when the entries $b_{i, j}(x)$ of $Q(x)$ belong to $L_{1}[0,1]$, that is, when there is not any condition about smoothness of the potential $Q(x)$ are obtained. Note that to obtain the asymptotic formulas of high accuracy by using the classical asymptotic expansions for the solutions of the matrix equation $-Y^{\prime \prime}+Q(x) Y=\lambda Y$ it is required that $Q(x)$ be differentiable (see [1-4]). To obtain the asymptotic formulas we consider the operator $L_{t}(Q)$ as perturbation of $L_{t}(C)$, where $C=\int_{0}^{1} Q(x) d x$, by $Q(x)-C$, that is, we take the operator $L_{t}(C)$ for an unperturbed operator and the operator of multiplication by $Q(x)-C$ for a perturbation. Therefore first we analyze the eigenvalues and eigenfunction of $L_{t}(C)$.

The adjoint operator to $L_{t}(Q)$ is $L_{t}\left(Q^{*}\right)$, where $Q^{*}(x)$ is the adjoint matrix to $Q(x)$. Since $Q(x)$ is Hermitian matrix, that is, $Q^{*}(x)=Q(x)$ and the boundary conditions (2) are self-adjoint the operators $L_{t}(Q), L_{t}(C)$ and $L_{t}(0)$ are self-adjoint. The eigenvalues of $C$, counted with multiplicity, and the corresponding orthonotmal eigenvectors are denoted by

$\mu_{1} \leq \mu_{2} \leq \ldots \leq \mu_{m}$ and $v_{1}, v_{2}, \ldots, v_{m}$ respectively. Thus

$$
C v_{j}=\mu_{j} v_{j},\left\langle v_{i}, v_{j}\right\rangle=\delta_{i, j}
$$

In these notations the eigenvalues and eigenfunctions of $L_{t}(C)$ are

$$
\begin{gathered}
\mu_{k, j}(t)=(2 \pi k+t)^{2}+\mu_{j}, \Phi_{k, j, t}(x)=v_{j} e^{i(2 \pi k+t) x}, \text { that is }, \\
\left(L(C)-\mu_{k, j}(t)\right) \Phi_{k, j, t}(x)=0 .
\end{gathered}
$$

To prove the asymptotic formulas for the eigenvalues $\lambda_{k, j}(t)$ and for the corresponding normalized eigenfunctions $\Psi_{k, j, t}(x)$ of $L_{t}(Q)$ we use the formula

$$
\left(\lambda_{k, j}(t)-\mu_{n, i}(t)\right)\left(\Psi_{k, j, t}, \Phi_{n, i, t}\right)=\left((Q(x)-C) \Psi_{k, j, t}, \Phi_{n, i, t}\right)
$$


for $n \in A(k, t)$, connecting the eigenvalues and eigenfunctions of the operators $L_{t}(Q)$ and $L_{t}(C)$, which can be obtained from

$$
L(Q(x)) \Psi_{k, j, t}(x)=\lambda_{k, j}(t) \Psi_{k, j, t}(x)
$$

by multiplying both sides by $\Phi_{n, i, t}(x)$ and using (11). Then we estimate the right-hand side of (12) ( see Lemma 2) by using Lemma 1. At last using the connecting formula (12) and lemmas 2, 3 we find the asymptotic formulas for the eigenvalues and eigenfunctions of $L_{t}(Q)$ ( see Theorem 1). Then using these asymptotic formulas, we find the conditions on the eigenvalues of the matrix $C$ for which the number of the gaps in the spectrum of the Hill's operator $L(Q)$ is finite. This result for differentiable $Q(x)$ is obtained in [4,5]. To estimate the right-hand side of (12) we use (7), (8), the following lemma, and the formula

$$
\left(\lambda_{k, j}(t)-(2 \pi n+t)^{2}\right)\left(\Psi_{k, j, t}, \varphi_{n, s, t}\right)=\left(\Psi_{k, j, t}, Q(x) \varphi_{n, s, t}\right),
$$

which can be obtained from (13) by multiplying both sides by $\varphi_{n, s, t}(x)$ and using $L_{t}(0) \varphi_{n, s, t}(x)=(2 \pi n+t)^{2} \varphi_{n, s, t}(x)$.

Lemma 1 For the right-hand side of (14) the followings hold:

$$
\begin{gathered}
\left(\Psi_{k, j, t}(x), Q(x) \varphi_{n, s, t}(x)\right)=\sum_{\substack{q=1,2, \ldots m \\
p=-\infty, \ldots, \infty}} b_{s, q, n-p}\left(\Psi_{k, j, t}, \varphi_{p, q, t}\right), \\
\left|\left(\Psi_{k, j, t}(x), Q(x) \varphi_{n, s, t}(x)\right)\right|<c_{4}
\end{gathered}
$$

for $n \in Z ;|k| \geq N ; s, j=1,2, \ldots, m$, where

$$
b_{s, q, n-p}=\int_{0}^{1} b_{s, q}(x) e^{2 \pi i(p-n) x} d x .
$$

Proof. Since $Q(x) \Psi_{k, j, t}(x) \in L_{1}^{m}[0,1]$ we have

$$
\lim _{n \rightarrow \infty}\left(Q(x) \Psi_{k, j, t}(x), \varphi_{n, s, t}(x)\right)=0, \forall s=1,2, \ldots, m .
$$

Therefore there exists a positive constant $C(k, j)$ and indices $k_{0}, j_{0}$ satisfying

$$
\max _{\substack{n \in \mathbb{Z}, s=1,2, \ldots, m}}\left|\left(\Psi_{k, j, t}, Q(x) \varphi_{n, s, t}\right)\right|=\left|\left(\Psi_{k, j, t}, Q(x) \varphi_{k_{0}, j_{0}, t}\right)\right|=C(k, j)
$$

Using this, (14), (5), we obtain

$$
\left|\left(\Psi_{k, j, t}(x), \varphi_{p, s, t}(x)\right)\right| \leq \frac{C(k, j)}{\left|\lambda_{k, j}(t)-(2 \pi p+t)^{2}\right|}
$$

for $p \notin A(k, t)$ and $s, j=1,2, \ldots, m$. This and (7) imply that

$$
\sum_{p: p>d}\left|\left(\Psi_{k, j, t}(x), \varphi_{p, s, t}(x)\right)\right|<\frac{c_{3} C(k, l)}{d}
$$


where $d>2|k|$ and $t \in\left[-\frac{\pi}{2}, \frac{3 \pi}{2}\right)$. Therefore the decomposition of $\Psi_{k, j, t}(x)$ by the orthonormal basis $\left\{\varphi_{p, q, t}(x): p \in \mathbb{Z}, q=1,2, \ldots, m\right\}$ is of the form

$$
\Psi_{k, j, t}(x)=\sum_{\substack{p:|p| \leq d, q=1,2, \ldots, m}}\left(\Psi_{k, j, t}(x), \varphi_{p, q, t}(x)\right) \varphi_{p, q, t}(x)+g_{d}(x),
$$

where $\sup _{x \in[0,1]}\left|g_{d}(x)\right|<\frac{c_{3} C(k, l)}{d}$. Putting this in $\left(\Psi_{k, j, t}(x), Q(x) \varphi_{n, s, t}(x)\right)$ and tending $d$ to $\infty$, we obtain (15).

Now we prove (16). Using (20) in $\left(\Psi_{k, j, t}(x), Q(x) \varphi_{k_{0}, j_{0}, t}(x)\right)$, isolating the terms with multiplicands $\left(\Psi_{k, j, t}(x), \varphi_{p, i, t}(x)\right)$ for $p=k,-k,-(k+1)$;

$q=1,2, \ldots, m$, and tending $d$ to $\infty$,we obtain

$$
\begin{aligned}
\left(\Psi_{k, j, t}(x), Q(x) \varphi_{k_{0}, j_{0}, t}(x)\right)= & \sum_{\substack{p=k,-k,-(k+1) ; \\
q=1,2, \ldots, m}} b_{j_{0}, q, k_{0}-p}\left(\Psi_{k, j, t}(x), \varphi_{p, q, t}(x)\right)+ \\
& \sum_{\substack{p \neq k,-k,-(k+1) \\
q=1,2, \ldots, m}} b_{j_{0}, q, k_{0}-p}\left(\Psi_{k, j, t}(x), \varphi_{p, q, t}(x)\right) . \quad(21)
\end{aligned}
$$

Since

$$
\left|b_{j, i, s}\right| \leq \max _{p, q=1,2, \ldots, m} \int_{0}^{1}\left|b_{p, q}(x)\right| d x<c_{5}, \forall j, i, s
$$

( see (17)) it follows from (19), (8), (6) that

$$
\left.\sum_{\substack{p \neq k,-k,-(k+1) \\ q=1,2, \ldots, m}} b_{j_{0}, q, k_{0}-p}\left(\Psi_{k, j, t}, \varphi_{p, q, t}\right)=O\left(\frac{\ln |k|}{k}\right) C(k, l)\right) .
$$

Therefore taking into account that the absolute value of the first summation in the right-hand side of (21) is not greater than $3 m c_{5}$ ( see (22)), we conclude that $|C(k, l)|<4 m c_{5}$ ( see (18)) which means that (16) holds

Lemma 2 Let $\Psi_{k, j, t}(x)$ be any normalized eigenfunctions corresponding to the eigenvalue $\lambda_{k, j}(t)$ of $L_{t}(Q)$. Then

$$
\left(\Psi_{k, j, t}(x),(Q(x)-C) \Phi_{n, i, t}(x)\right)=O\left(\frac{\ln |k|}{k}\right)+O\left(b_{k}\right)
$$

for $n \in A(k, t)$ and $i=1,2, \ldots, m$, where

$$
b_{k}=\max \left\{\left|b_{i, j, n}\right|: i, j=1,2, \ldots m ; n=2 k,-2 k, 2 k+1,-2 k-1\right\} .
$$

In particular, for $t \in T(k)$ the formula

$$
\left(\Psi_{k, j, t}(x),(Q(x)-C) \Phi_{k, i, t}(x)\right)=O\left(\frac{\ln |k|}{k}\right)
$$

holds. The formula (23) is uniform with respect to $t \in\left[-\frac{\pi}{2}, \frac{3 \pi}{2}\right)$. 
Proof. Since $\Phi_{n, s, t}(x) \equiv v_{s} e^{i(2 \pi n+t) x}$, to prove (23) it is enough to show that

$$
\left(\Psi_{k, j, t}(x),(Q(x)-C) \varphi_{n, s, t}(x)\right)=O\left(\left(\frac{\ln |k|}{k}\right)+O\left(b_{k}\right)\right.
$$

for $s=1,2, \ldots, m$ and $n \in A(k, t)$. Using the obvious relation

$$
\left(\Psi_{k, j, t}(x), C \varphi_{n, s, t}(x)\right)=\sum_{q=1,2, \ldots, m} b_{s, q, 0}\left(\Psi_{k, j, t}(x), \varphi_{n, q, t}(x)\right)
$$

and (15), we see that

$$
\begin{gathered}
\left(\Psi_{k, j, t},(Q(x)-C) \varphi_{n, s, t}(x)\right)=\sum_{\substack{p: p \in A(k, t) \backslash n \\
q=1,2, \ldots, m}} b_{s, q, n-p}\left(\Psi_{k, j, t}(x), \varphi_{p, q, t}(x)\right)+ \\
\sum_{\substack{p: p \notin A(k, t) \\
q=1,2, \ldots, m}} b_{s, q, k-p}\left(\Psi_{k, j, t}(x), \varphi_{p, q, t}(x)\right) .
\end{gathered}
$$

On the other hand, it follows from (19), (18), (16) that

$$
\left|\left(\Psi_{k, j, t}(x), \varphi_{p, q, t}(x)\right)\right| \leq \frac{c_{4}}{\left|\lambda_{k, j}(t)-(2 \pi p+t)^{2}\right|}
$$

for $p \notin A(k, t)$ and $q=1,2, \ldots, m$. Using this, (22), and (8) we see that the second summation of the right-hand side of $(27)$ is $O\left(\left(\frac{\ln |k|}{k}\right)\right.$. Besides it follows from (6), (24) that the first summation of the right-hand side of (27) is $O\left(b_{k}\right)$, since for $n \in A(k, t)$ and $p \in A(k, t) \backslash n$ we have $n-p \in\{2 k,-2 k, 2 k+1,-2 k-1\}$. If $t \in T(k)$ then the first summation of the right-hand side of (27) is absent, since $A(k, t)=\{k\}$ and $A(k, t) \backslash n=\emptyset$ for $t \in T(k)$ and $n \in A(k, t)$ (see (6)). Thus (25) is also proved.

Lemma 3 For each eigenfunction $\Psi_{k, j, t}(x)$ of $L_{t}(Q)$ there exists an eigenfunction $\Phi_{n, i, t}(x)$ of $L_{t}(C)$ satisfying

$$
\left|\left(\Psi_{k, j, t}(x), \Phi_{n, i, t}(x)\right)\right|>c_{6}
$$

and for each eigenfunction $\Phi_{k, j, t}(x)$ of $L_{t}(C)$ there exists an eigenfunction $\Psi_{n, i, t}(x)$ of $L_{t}(Q)$ satisfying

$$
\left|\left(\Phi_{k, j, t}(x), \Psi_{n, i, t}(x)\right)\right|>c_{7},
$$

where $n \in A(k, t) ; i, j=1,2, \ldots, m$ and $|k| \geq N$.

Proof. It follows from (28) and (9) that

$$
\sum_{q=1,2, \ldots, m}\left(\sum_{n: n \notin A(k, t)}\left|\left(\Psi_{k, j, t}, \varphi_{n, q, t}\right)\right|^{2}\right)=O\left(\frac{(\ln |k|)^{2}}{k^{2}}\right) .
$$

Therefore taking into account that $\Phi_{n, s, t}(x)=v_{s} e^{i(2 \pi n+t) x}$, where $v_{s}$ for 
$s=1,2, \ldots m$ are the normalized eigenvector of $C$, we get

$$
\sum_{i=1,2, \ldots, m}\left(\sum_{n: n \notin A(k, t)}\left|\left(\Psi_{k, j, t}, \Phi_{n, i, t}\right)\right|^{2}\right)=O\left(\frac{(\ln |k|)^{2}}{k^{2}}\right) .
$$

Since $\left\{\Phi_{n, i, t}(x): i=1,2, \ldots, m ; n \in Z\right\}$ is an orthonormal basis in $L_{2}^{m}[0,1]$, we have

$$
\sum_{i=1,2, \ldots, m ; n \in A(k, t)}\left|\left(\Psi_{k, j, t}(x), \Phi_{n, i, t}(x)\right)\right|^{2}=1+O\left(\frac{(\ln |k|)^{2}}{k^{2}}\right)
$$

Now taking into account that the number of the orthonormal eigenfunctions $\Phi_{n, i, t}(x)$ for $n \in A(k, t), i=1,2, \ldots, m$ less than $2 m$ we obtain the proof of (29). Instead of (9) using (10), taking into account that the eigenfunctions of the operator $L_{t}(Q)$ form an orthonormal basis in $L_{2}^{m}(0,1)$, and arguing as in the proof of (33) we get

$$
\begin{gathered}
\sum_{i=1,2, \ldots, m}\left(\sum_{n: n \notin A(k, t)}\left|\left(\varphi_{k, j, t}, \Psi_{n, i, t}\right)\right|^{2}\right)=O\left(\frac{(\ln |k|)^{2}}{k^{2}}\right) \\
\sum_{i=1,2, \ldots, m}\left(\sum_{n: n \notin A(k, t)}\left|\left(\Phi_{k, j, t}, \Psi_{n, i, t}\right)\right|^{2}\right)=O\left(\frac{(\ln |k|)^{2}}{k^{2}}\right), \\
\sum_{i=1,2, \ldots, m}\left(\sum_{n: n \in A(k, t)}\left|\left(\Phi_{k, j, t}, \Psi_{n, i, t}\right)\right|^{2}\right)=1+O\left(\frac{(\ln |k|)^{2}}{k^{2}}\right)
\end{gathered}
$$

and the proof of $(30)$

Theorem 1 (a) All big eigenvalues of $L_{t}(Q(x))$ lie in $\varepsilon_{k} \equiv c_{8}\left(\left|\frac{\ln |k|}{k}\right|+b_{k}\right)$ neighborhood $U\left(\varepsilon_{k}, \mu_{k, i}\right)$ of the eigenvalues $\mu_{k, i}(t)=(2 \pi k+t)^{2}+\mu_{i}$ for $|k| \geq N$, $i=1,2, \ldots, m$ of $L_{t}(C)$. Moreover, for each big eigenvalues $\mu_{k, j}(t)$ of $L_{t}(C)$ there exists an eigenvalue of $L_{t}(Q(x))$ lying in $U\left(\varepsilon_{k}, \mu_{k, j}\right)$.

(b) If $t \neq 0, \pi$ then the eigenvalues of $L_{t}(Q)$ consist of $m$ sequences (3) satisfying

$$
\lambda_{k, j}(t)=(2 \pi k+t)^{2}+\mu_{j}+O\left(\frac{(\ln |k|)}{k}\right),
$$

where $\mu_{1} \leq \mu_{2} \leq \ldots \leq \mu_{m}$ are the eigenvalues of $C$, and any normalized eigenfunction $\Psi_{k, j, t}(x)$ corresponding to $\lambda_{k, j}$ obey

$$
\left\|\Psi_{k, j, t}(x)-P \Psi_{k, j, t}(x)\right\|=O\left(\frac{(\ln |k|)}{k}\right),
$$

where $P$ is the orthogonal projection onto the eigenspace corresponding to $\mu_{k, j}$.

(c) If $\mu_{j}$ is a simple eigenvalue of the matrix $C$ and $t \notin B\left(\alpha_{k}, \mu_{j}\right)$, where

$$
B\left(\alpha_{k}, \mu_{j}\right)=\bigcup_{\substack{n=0,1 ; \\ p=0,1, \ldots, m}}\left\{\pi n+\frac{\mu_{p}-\mu_{j}-\alpha_{k}}{4 \pi(2 k+n)}, \pi n+\frac{\mu_{p}-\mu_{j}+\alpha_{k}}{4 \pi(2 k+n)}\right\},
$$


$k \geq N$ and $\alpha_{k}=\sqrt{\varepsilon_{k}}$, then there exists a unique eigenvalue, denoted by $\lambda_{k, j}(t)$, of $L_{t}(Q(x))$ lying in $U\left(\varepsilon_{k}, \mu_{k, j}\right)$. This eigenvalue is a simple eigenvalue of $L_{t}(Q(x))$ and the corresponding normalized eigenfunction $\Psi_{k, j, t}(x)$ satisfy

$$
\Psi_{k, j, t}(x)=v_{j} e^{i(2 \pi k+t) x}+O\left(\alpha_{k}\right),
$$

where $v_{j}$ is the normalized eigenvector of $C$ corresponding to the eigenvalue $\mu_{j}$.

(d) Suppose that there exists at least three simple eigenvalues $\mu_{j_{1}}<\mu_{j_{2}}<\mu_{j_{3}}$ of $C$ and the set $\left\{\mu_{j_{p}}+\mu_{i_{p}}: p=1,2,3\right\}$ contains two points for each triple $\left(i_{1}, i_{2}, i_{3}\right)$, where $i_{1}, i_{2}, i_{3}=1,2, \ldots, m$. Then there exist a number $H$ such that $(H, \infty) \in S(L)$, that is, the number of the gaps in the spectrum of $L(Q)$ is finite.

Proof. (a) Dividing both sides of (12) by $\left(\Psi_{k, j, t}(x), \Phi_{n, i, t}(x)\right)$ and using (23), (29) we get the proof of the first statement of $(a)$. Using (30), in the same way, we obtain the proof of the second statement of $(a)$.

(b). If $t \neq 0, \pi$ then there exist $N$ such that $t \in T(k)$ for $k>N$. Therefore instead of (23) using (25), taking into account that $A(k, t)=\{k\}$ for $t \in T(k)$, and repeating the proof of $(a)$ we see that the eigenvalues $\lambda_{k, j}$ for $j=1,2, \ldots, m$ of $L_{t}(Q(x))$ lie in $U\left(\delta_{k}, \mu_{k, i}\right)$ for $i=1,2, \ldots, m$ and for each eigenvalue $\mu_{k, i}$ of $L_{t}(C)$ there exists an eigenvalue of $L_{t}(Q(x))$ lying in $U\left(\delta_{k}, \mu_{k, i}\right)$, where $|k| \geq N$, $\delta_{k}=c_{9} \frac{\ln |k|}{|k|}$.

Now we prove that if the multiplicity of the eigenvalue $\mu_{j}$ of $C$ is $q$ then there exist precisely $q$ eigenvalues of the operator $L_{t}(Q)$ lying in $U\left(\delta_{k}, \mu_{k, j}\right)$ for $|k| \geq N$. The eigenvalues of $L_{t}(Q)$ and $L_{t}(C)$ can be numbered in the following way $\lambda_{k, 1} \leq \lambda_{k, 2} \leq \ldots \leq \lambda_{k, m}$ and $\mu_{k, 1} \leq \mu_{k, 2} \leq \ldots \leq \mu_{k, m}$. If the matrix $C$ has $r$ different eigenvalues $\mu_{j_{1}}, \mu_{j_{2}}, \ldots, \mu_{j_{r}}$, where $j_{1}<j_{2}<\ldots<j_{r}=m$, with multiplicities $j_{1}, j_{2}-j_{1}, \ldots, j_{r}-j_{r-1}$ then we have

$$
\begin{aligned}
\mu_{j_{1}} & <\mu_{j_{2}}<\ldots<\mu_{j_{r}} ; \mu_{1}=\mu_{2}=\ldots=\mu_{j_{1}} \\
\mu_{j_{1}+1} & =\mu_{j_{1}+2}=\ldots=\mu_{j_{2}} ; \ldots ; \mu_{j_{r-1}+1}=\mu_{j_{r-2}+2}=\ldots=\mu_{j_{r}} .
\end{aligned}
$$

Suppose that there exist precisely $s_{1}, s_{2}, \ldots, s_{r}$ eigenvalues of $L_{t}(Q(x))$ lying in neighborhoods $U\left(\delta_{k}, \mu_{k, j_{1}}\right), U\left(\delta_{k}, \mu_{k, j_{2}}\right), \ldots, U\left(\delta_{k}, \mu_{k, j_{r}}\right)$ respectively. Since

$\delta_{k}<\frac{1}{2} \min _{p=1,2, \ldots, r-1}\left|\mu_{j_{p}+1}-\mu_{j_{p}}\right|$ for $|k| \geq N$ these neighborhoods are pairwise disjoints. Thus in the first paragraph of the proof of $(b)$ we have proved that

$$
s_{1}+s_{2}+\ldots+s_{r}=m
$$

Now we prove that $s_{1}=j_{1}, s_{2}=j_{2}-j_{1}, \ldots, s_{r}=j_{r}-j_{r-1}$. Due to the notations the eigenvalues $\lambda_{k, 1}, \lambda_{k, 2} \ldots, \lambda_{k, s_{1}}$ of the operator $L_{t}(Q)$ lie in $U\left(\delta_{k}, \mu_{k, 1}\right)$ and

$$
\left|\lambda_{k, j}-\mu_{k, i}\right|>\frac{1}{2} \min _{p: p>j_{1}}\left|\mu_{1}-\mu_{p}\right|
$$

for $j \leq s_{1}$ and $i>j_{1}$. Therefore it follows from

$$
\left(\lambda_{k, j}(t)-\mu_{k, i}\right)\left(\Psi_{k, j, t}, \Phi_{k, i, t}\right)=\left((Q(x)-C) \Psi_{k, j, t}(x), \Phi_{k, i, t}(x)\right)
$$


and (25) that

$$
\left.\sum_{i>j_{1}}\left|\left(\Psi_{k, j, t}, \Phi_{k, i, t}\right)\right|^{2}\right)=O\left(\frac{(\ln |k|)^{2}}{k^{2}}\right), \forall j \leq s_{1}
$$

Using this and (33), and taking into account that $A(k, t)=\{k\}$ for $t \in T(k)$ ( see (6)), we conclude that there exists normalized eigenfunction corresponding to $\mu_{k, 1}=\mu_{k, 2}=\ldots=\mu_{k, j_{1}}$ and denoted by $\Phi_{k, j, t}(x)$ such that

$$
\Psi_{k, j, t}(x)=\Phi_{k, j, t}(x)+O\left(\frac{\ln |k|}{k}\right)
$$

for $j \leq s_{1}$. Since $\Psi_{k, 1, t}(x), \Psi_{k, 2, t}(x), \ldots, \Psi_{k, s_{1}, t}(x)$ are orthonormal system we have

$$
\left(\Phi_{k, j, t}(x), \Phi_{k, i, t}(x)\right)=\delta_{i, j}+O\left(\frac{\ln |k|}{k}\right)
$$

for $i, j=1,2, \ldots, s_{1}$. This formula imply that the dimension $j_{1}$ of the eigenspace of $L_{t}(C)$ corresponding to the eigenvalue $\mu_{k, 1}=\mu_{1}+(2 \pi k+t)^{2}$ is not less than $s_{1}$. Thus $s_{1} \leq j_{1}$. In the same way we prove that $s_{2} \leq j_{2}-j_{1}, \ldots, s_{r} \leq j_{r}-j_{r-1}$. These inequalities with the equalities (41), $j_{r}=m$ imply that $s_{1}=j_{1}$,

$s_{2}=j_{2}-j_{1}, \ldots, s_{r}=j_{r}-j_{r-1}$. Therefore taking into account that ( see $(3),(4))$ the eigenvalues of the operator $L_{t}(Q)$ consist of $m$ sequences satisfying (4) we get the prove of (36). The proof of (37) follows from (44).

(c) To consider the simplicity of $\mu_{k, j}(t)$ and $\lambda_{k, j}(t)$ for $k \geq N$ we introduce the set

$$
S(k, j, n, i)=\left\{t \in\left[-\frac{\pi}{2}, \frac{3 \pi}{2}\right):\left|\mu_{k, j}(t)-\mu_{n, i}(t)\right|<\alpha_{k}\right\}
$$

for $(n, i) \neq(k, j)$. It follows from (5) that $S(k, j, n, i)=\emptyset$ for $n \neq k,-k,-k-1$. If $\mu_{j}$ is a simple eigenvalue of the matrix $C$ then $S(k, j, k, i)=\emptyset$ for $i \neq j$. It remains to consider the sets $S(k, j,-k, i), S(k, j,-k-1, i)$ By direct calculations of the differences $\mu_{k, j}(t)-\mu_{-k, i}(t), \mu_{k, j}(t)-\mu_{-k-1, i}(t)$ one can easily verify that

$$
\begin{gathered}
S(k, j,-k, i)=\left(\frac{\mu_{i}-\mu_{j}-\alpha_{k}}{8 \pi k}, \frac{\mu_{i}-\mu_{j}+\alpha_{k}}{8 \pi k}\right) \\
S(k, j,-k-1, i)=\left(\pi+\frac{\mu_{i}-\mu_{j}-\alpha_{k}}{4 \pi(2 k+1)}, \pi+\frac{\mu_{i}-\mu_{j}+\alpha_{k}}{4 \pi(2 k+1)}\right)
\end{gathered}
$$

These relations with (38) imply that

$$
B\left(\alpha_{k}, \mu_{j}\right)=\bigcup_{\substack{n \in \mathbb{Z} ;(n, i) \neq(k, j) \\ i=0,1, \ldots, m}} S(k, j, n, i)=\bigcup_{\substack{n=-k,-k-1 \\ i=0,1, \ldots, m}} S(k, j, n, i)
$$

Therefore it follows from the definition of $S(k, j, n, i)$ ( see (45)) that if

$t \notin B\left(\alpha_{k}, \mu_{j}\right)$, then

$$
\left|\mu_{k, j}(t)-\mu_{n, i}(t)\right| \geq \alpha_{k}
$$


for all $(n, i) \neq(k, j)$. Hence $\mu_{k, j}(t)$ is a simple eigenvalue for $t \notin B\left(\alpha_{k}, \mu_{j}\right)$. By (a) there exist an eigenvalue of $L_{t}(Q)$ lying in $U\left(\varepsilon_{k}, \mu_{k, j}\right)$. Denote this eigenvalue by $\lambda_{k, j}(t)$. Thus $\lambda_{k, j}$ is an eigenvalue of $L_{t}(Q(x))$ satisfying

$$
\left|\lambda_{k, j}(t)-\mu_{k, j}(t)\right|<\varepsilon_{k} .
$$

It follows from this and (49) that

$$
\left|\lambda_{k, j}(t)-\mu_{n, i}(t)\right|>\frac{1}{2} \alpha_{k}
$$

for $n \in A(k, t), i=1,2, \ldots, m$, and $(n, i) \neq(k, j)$ since $k \geq N, \alpha_{k}=\sqrt{\varepsilon_{k}}$ and $\varepsilon_{p} \rightarrow 0$ as $p \rightarrow \infty$. Let $\Psi_{k, j, t}(x)$ be any normalized eigenfunction corresponding to $\lambda_{k, j}$. Using (12), (23), definition of $\varepsilon_{k}$, and (51), we obtain

$$
\left(\Psi_{k, j, t}(x), \Phi_{n, i, t}(x)\right)=O\left(\alpha_{k}\right)
$$

for $(n, i) \neq(k, j)$ and $n \in A(k, t)$. This and (35) imply that $\Psi_{k, j, t}(x)$ satisfies (39). Thus we have proved that (39) holds for any normalized eigenfunction corresponding to any eigenvalue lying in $U\left(\varepsilon_{k}, \mu_{k, j}\right)$. If there are two different eigenvalue or a multiple eigenvalue lying in $U\left(\varepsilon_{k}, \mu_{k, j}\right)$ then there are two orthonormal eigenfunction satisfying (39) which is impossible. Hence there exists unique eigenvalue $\lambda_{k, j}(x)$ lying in $U\left(\varepsilon_{k}, \mu_{k, j}\right)$ and this is a simple eigenvalue.

(d) In (c) we proved that if $k \geq N, t \notin B\left(\alpha_{k}, \mu_{j_{p}}\right)$, where $p=1,2,3$, then there exists a unique eigenvalue of $L_{t}(Q)$ lying in $U\left(\varepsilon_{k}, \mu_{k, j_{p}}\right)$. We denoted this unique eigenvalue by $\lambda_{k, j_{p}}(t)$ and proved that it it a simple eigenvalue. Let us prove that $\lambda_{k, j_{p}}(t)$ is a continuous function at $t_{0} \in\left[-\frac{\pi}{2}, \frac{3 \pi}{2}\right) \backslash B\left(\alpha_{k}, \mu_{j_{p}}\right)$. Since $\lambda_{k, j_{p}}\left(t_{0}\right)$ is a simple eigenvalue it is a simple root of the characteristic determinant $\Delta(\lambda, t)$ of the operator $L_{t}(Q)$. Therefore there exists a neighborhood $U\left(t_{0}\right)$ of $t_{0}$ and a continuous in $U\left(t_{0}\right)$ function $\Lambda(t)$ such that $\Lambda\left(t_{0}\right)=\lambda_{k, j_{p}}\left(t_{0}\right), \Lambda(t)$ is an eigenvalue of $L_{t}(Q)$ for $t \in U\left(t_{0}\right)$ and

$$
\left|\Lambda(t)-\mu_{k, j_{p}}(t)\right|<\varepsilon_{k}, \forall t \in U\left(t_{0}\right),
$$

since $\left|\Lambda\left(t_{0}\right)-\mu_{k, j_{p}}\left(t_{0}\right)\right|<\varepsilon_{k}$ and the functions $\Lambda(t), \mu_{k, j_{p}}(t)$ are continuous. Now taking into account that there exists unique eigenvalue of $L_{t}(Q)$ lying in $U\left(\varepsilon_{k}, \mu_{k, j_{p}}\right)$, we obtain that $\Lambda(t)=\lambda_{k, j_{p}}(t)$ for $t \in U\left(t_{0}\right)$, and hence $\lambda_{k, j_{p}}(t)$ is continuous at $t_{0}$. Now we prove that there exists $H$ such that

$$
(H, \infty) \subset\left\{\lambda_{k, j_{p}}(t): t \in\left[-\frac{\pi}{2}, \frac{3 \pi}{2}\right) \backslash B\left(\alpha_{k}, \mu_{j_{p}}\right), k=N, N+1, \ldots,\right\} .
$$

It is clear that

$$
(h, \infty) \subset\left\{\mu_{k, j_{p}}(t): t \in\left[-\frac{\pi}{2}, \frac{3 \pi}{2}\right), k=N, N+1, \ldots,\right\},
$$

where $h=\mu_{N, j_{3}}\left(-\frac{\pi}{2}\right)$. Since $\mu_{k, j_{p}}(t)=(2 \pi k+t)^{2}+\mu_{j_{p}}$ is increasing function for $k \geq N$, it follows from (48), (47), (46) that

$$
\left\{\mu_{k, j_{p}}(t): t \in B\left(\alpha_{k}, \mu_{j_{p}}\right)\right\} \subset \bigcup_{\substack{n=0,1 ; \\ i=0,1, \ldots, m}} C\left(k, j_{p}, i, \alpha_{k}, n\right),
$$


where $C\left(k, j_{p}, i, \alpha_{k}, n\right)=\left\{x \in \mathbb{R}:\left|x-(\pi(2 k+n))^{2}-\frac{\mu_{i}+\mu_{j_{p}}}{2}\right|<\alpha_{k}\right\}$. Therefore

$$
\left\{\mu_{k, j_{p}}(t): t \in\left[-\frac{\pi}{2}, \frac{3 \pi}{2}\right) \backslash B\left(\alpha_{k}, \mu_{j_{p}}\right), k \geq N\right\} \supset(h, \infty) \backslash \underset{\substack{n=0,1 ; k \geq N \\ i=0,1, \ldots, m}}{\bigcup} C\left(k, j_{p}, i, \alpha_{k}, n\right)
$$

Now using (50) and the continuity of $\lambda_{k, j_{p}}(t)$ on $\left[-\frac{\pi}{2}, \frac{3 \pi}{2}\right) \backslash B\left(\alpha_{k}, \mu_{j_{p}}\right)$ we get

$$
\left\{\lambda_{k, j_{p}}(t): t \in\left[-\frac{\pi}{2}, \frac{3 \pi}{2}\right) \backslash B\left(\alpha_{k}, \mu_{j_{p}}\right), k \geq N\right\} \supset(H, \infty) \backslash \bigcup_{\substack{n=0,1 ; k \geq N \\ i=0,1, \ldots, m}} C\left(k, j_{p}, i, 2 \alpha_{k}, n\right),
$$

where $H=h+1$. Thus

$$
\bigcup_{p=1,2,3}\left((H, \infty) \backslash \bigcup_{n=0,1 ; k \geq N ; i=0,1, \ldots, m} C\left(k, j_{p}, i, 2 \alpha_{k}, n\right)\right) \subset S(L)
$$

To prove that $(H, \infty) \subset S(L)$ it is enough to show that the set

$$
\bigcap_{p=1,2,3}\left(\bigcup_{n=0,1 ; k \geq N ; i=0,1, \ldots, m} C\left(k, j_{p}, i, 2 \alpha_{k}, n\right)\right)
$$

is empty. If this set contains an element $x$, then

$$
x \in \bigcup_{n=0,1 ; k \geq N ; i=0,1, \ldots, m} C\left(k, j_{p}, i, 2 \alpha_{k}, n\right)
$$

for all $p=1,2,3$. Using this and the definition of $C\left(k, j_{p}, i, 2 \alpha_{k}, n\right)$, we obtain that there exist $k \geq N ; n=0,1$ and $i_{p}$ such that

$$
\left|x-(\pi(2 k+n))^{2}-\frac{\mu_{j_{p}}+\mu_{i_{p}}}{2}\right|<2 \alpha_{k}
$$

for all $p=1,2,3$ and hence $\left|\frac{\mu_{j_{q}}+\mu_{i_{q}}}{2}-\frac{\mu_{j_{p}}+\mu_{i_{p}}}{2}\right|<4 \alpha_{k}$ for all $p, q=1,2,3$. Since $\alpha_{k} \rightarrow 0$ as $k \rightarrow \infty$ the last inequality imply that $\mu_{j_{1}}+\mu_{i_{1}}=\mu_{j_{2}}+\mu_{i_{2}}=\mu_{j_{3}}+\mu_{i_{3}}$ which contradicts the condition of $(d)$.

\section{References}

[1] M. A. Naimark, Linear Differential Operators, George G. Harap\&Company, London, 1967.

[2] R. Carlson, Large Eigenvalues and Trace Formulas for Matrix SturmLiouville Problems, SIAM Journal on Mathematical Analysis, vol. 30 (1999) no. 5, 949-962.

[3] Maksudov, F.G., Veliev O.A., Nonself-adjoint Differential Operators in the Space of Vector Function with Periodic Coefficients, Soviet Math. Dokl., 23 (1981), 475-478. 
[4] Maksudov, F.G., Veliev O.A., Spectral Analysis of Differential Operators with Periodic Matrix Coefficients. Differential Equations+, vol. 25 (1989), no.3, 271-277.

[5] O.A. Veliev, On the Spectrum of the Schrodinger Operator with Periodic Potential, Dokl. Akad. Nauk SSSR 268 (1983), no 6, 1289-1292. 